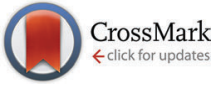

Cite this: Phys. Chem. Chem. Phys., 2016, 18, 15988

Received 10th January 2016, Accepted 10th May 2016

DOI: $10.1039 / c 6 c p 00178 \mathrm{e}$

www.rsc.org/pccp

\title{
Secondary phases and their influence on the composition of the kesterite phase in CZTS and CZTSe thin films $\dagger$
}

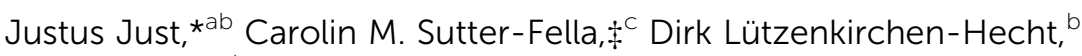 \\ Ronald Frahm, ${ }^{\text {b }}$ Susan Schorr ${ }^{a}$ and Thomas Unold ${ }^{* a}$
}

\begin{abstract}
Secondary phases zinc sulfide/selenide and copper sulfide in $\mathrm{Cu}_{2} \mathrm{ZnSnS}_{4}$ (CZTS) and $\mathrm{Cu}_{2} \mathrm{ZnSnSe}_{4}$ (CZTSe) thin film samples are investigated by $\mathrm{X}$-ray absorption near edge structure (XANES) analysis at the chalcogen $\mathrm{K}$-edges. Because of the formation of secondary phases the composition of the kesterite phase can deviate significantly from the total sample composition. For a large set of non-stoichiometric samples we find that the cation ratios of the kesterite phase never exceed $Z n / S n=1$ even for $Z n$-rich CZTS and CZTSe, with all excess Zn being contained in secondary phases. For CZTS the cation ratios are found to be additionally constrained by $\mathrm{Cu} / \mathrm{Sn} \leq 2$, which means that $\mathrm{Cu}$-excess always leads to the formation of $\mathrm{Cu}_{x} \mathrm{~S}$ secondary phases. These results give clear bounds on the $\mathrm{Cu}$-rich and $\mathrm{Zn}$-rich sides of the single phase region in polycrystalline CZTS/Se thin films.
\end{abstract}

\section{Introduction}

Multinary chalcogenide materials have high potential for optoelectronic applications, because of their widely tunable optical and electronic properties. ${ }^{1,2}$ For example, $\mathrm{Cu}_{2} \mathrm{ZnSnS}_{4}, \mathrm{Cu}_{2} \mathrm{ZnSnSe}_{4}$ and solid solutions thereof (CZTSSe) can be applied as absorbers in thin film solar cells, due to their p-type semiconducting nature and an optical band gap between 1 and $1.5 \mathrm{eV}$ which allows an optimal match of the absorption properties to the sun spectrum. $^{3-6}$

However, as the possibility of multiple phase formation increases with the number of constituent elements in these compounds, the identification and quantification of secondary phases become a crucial issue. A number of techniques have been applied for secondary phase identification in CZTS(e) thin films such as X-ray diffraction (XRD), ${ }^{7,8}$ microprobe fluorescence analysis, Raman spectroscopy, ${ }^{9-13}$ and X-ray absorption near

\footnotetext{
${ }^{a}$ Department Structure and Dynamics of Energy Materials, Helmholtz-Zentrum Berlin für Materialien und Energie GmbH, Hahn-Meitner Platz 1, 14109 Berlin, Germany. E-mail: justus.just@helmholtz-berlin.de, unold@helmholtz-berlin.de

${ }^{b}$ Fakultät 4 - Physik, Bergische Universität Wuppertal, Gaussstrasse 20, 42119 Wuppertal, Germany

${ }^{c}$ Laboratory for Thin Films and Photovoltaics, Empa - Swiss Federal Laboratories for Materials Science and Technology, Ueberlandstrasse 129, 8600 Duebendorf, Switzerland

$\dagger$ Electronic supplementary information (ESI) available. See DOI: 10.1039/ c6cp00178e

\$ Present address: Electrical Engineering and Computer Sciences, University of California, Berkeley, California 94720, USA.
}

edge spectroscopy (XANES). ${ }^{14}$ Some of the most important secondary phases in CZTS(e) are ZnS/ZnSe, which easily form because of their large negative formation enthalpies, ${ }^{15}$ in particular under Zn-rich growth conditions which have been employed in most experimental studies. ${ }^{5,16-19}$ At the same time $\mathrm{ZnS}$ and ZnSe are very difficult to be detected by room temperature XRD, because their diffraction peaks overlap with the peaks of the CZTS and CZTSe main phases. ${ }^{7}$ While $\mathrm{ZnS}$ and $\mathrm{ZnSe}$ can be detected by UV-Raman analysis, ${ }^{10}$ this technique is very surface sensitive and so far does not allow a quantitative determination of the phase content. Recently it has been shown that ZnS in CZTS can be unambiguously identified and even quantified by XANES measured at the K-edge of sulfur. ${ }^{14}$ In the present study we will show that the same analysis is also possible for ZnSe in CZTSe.

Quantification of the secondary phase content is important for the following reasons: it has been found in numerous studies that the electronic properties of kesterite-based solar cells correlate with the sample composition. ${ }^{2,20-22}$ However, in all of these studies the presence of secondary phases is not taken account of, which means that in reality the given correlations do not address the actual composition of the CZTS(e) phase itself. For instance, if $\mathrm{ZnS}$ is present in $\mathrm{Zn}$-rich CZTS samples, the $\mathrm{Cu} /(\mathrm{Zn}+\mathrm{Sn})$-ratio of the sample may be $\mathrm{Cu}$-poor, while the $\mathrm{Cu} /(\mathrm{Zn}+\mathrm{Sn})$ ratio of the kesterite phase is in fact larger than 1 , if the $\mathrm{ZnS}$ phase is properly accounted for.

In the present study we will present a method which allows the determination of the real composition of the CZTS (CZTSe) phase by a combination of X-ray fluorescence spectroscopy (XRF) and XANES measurements at the chalcogen K-edges. 
Analyzing a large series of samples this method allows us to deduce the existence region of single phase kesterite in the quasi-ternary phase diagrams with respect to $\mathrm{Zn}$-rich conditions (Se and S system) and Cu-rich conditions (S system). As there are very few experimental phase diagrams available for $\mathrm{CZTS}^{23-25}$ and to our knowledge none for CZTSe, these results are expected to provide a further understanding of the role of secondary phases in kesterite materials.

\section{Experimental section}

CZTS thin film samples of various compositions with different $\mathrm{Cu} / \mathrm{Sn}$ and $\mathrm{Zn} / \mathrm{Sn}$-ratios as well as a $\mathrm{Cu}_{x} \mathrm{~S}$ reference sample were prepared by physical vapor deposition using single stage co-evaporation of copper, zinc sulfide and tin at a nominal substrate temperature of $550{ }^{\circ} \mathrm{C}^{26}$ Molybdenum-coated soda lime glass was used as a substrate. Elemental compositions were measured by micro-X-ray fluorescence (XRF) mapping and averaging over the sample area, revealing no significant lateral inhomogeneities. Copper and zinc K-emission lines as well as Sn L-emission lines were used for the evaluation of the composition in order to avoid a contribution of the Sn-containing backside of the glass substrate, as it would be the case if the Sn $\mathrm{K}$-line is evaluated. To obtain accurate sample compositions, self-absorption effects have been taken into account based on calculations using the PyMCA software tool. ${ }^{27}$ XRF measurements were calibrated with CZTS thin film reference samples of known composition, which have been investigated by X-ray absorption spectroscopy and have been directly compared with Rutherford backscattering measurements. ${ }^{28}$ The X-ray absorption near edge structure (XANES) of the K-edge of sulfur $(2472 \mathrm{eV})$ was measured at Beamline A1 at the DORIS synchrotron at $\mathrm{HASYLAB}^{29}$ in a transmission setup. For measurement details, refer to a recent publication. ${ }^{14}$ All sulfur K-edge spectra are background subtracted and normalized using a pre-edge region from $2447.6 \mathrm{eV}$ to $2464.3 \mathrm{eV}$ and a post-edge region of 31-93 eV above the edge.

CZTSSe thin films were prepared by non-vacuum solution processing by dissolving the metal precursors $\mathrm{SnCl}_{2}(98 \%$ from Sigma-Aldrich), $\mathrm{ZnCl}_{2}$ (99.99\% from Alfa Aesar), and $\mathrm{CuCl}_{2}$ (98\%+ from Alfa Aesar) in dimethyl sulfoxide (DMSO, 99.9\% from Alfa Aesar), and adding thiourea $(99 \%+$ from SigmaAldrich). ${ }^{30,31}$ Precursor samples were prepared by iterating cycles of spin-coating and drying on a hot plate at $320{ }^{\circ} \mathrm{C}$ under atmospheric conditions using Mo-coated soda-lime glass substrates. In order to vary the sample composition along the $\mathrm{ZnSe}-\mathrm{Cu}_{2} \mathrm{SnSe}_{3}$-tie line, the $\mathrm{Zn} / \mathrm{Sn}$-ratios of the precursors were set within $0.5<\mathrm{Zn} / \mathrm{Sn}<3.8$. $\mathrm{Cu}-\mathrm{Zn}-\mathrm{Sn}-\mathrm{S}$ precursor samples were converted to CZTSSe by sintering at $560{ }^{\circ} \mathrm{C}$ for $25 \mathrm{~min}$ in elemental Se atmosphere. Due to an infinite supply of selenium during the sintering process, nearly all sulfur contained in the precursor was replaced by selenium. After selenization no significant amount of sulfur was found in the CZTSSe solid solution samples as the (112)-diffraction peak was measured to be at the position expected for the pure CZTSe material.
Thus, in the following these samples are denoted CZTSe. The compositional analysis of the CZTSe samples was performed by EDX (20 kV acceleration voltage) from the top view. The penetration volume of the electron beam at this energy is about the full film thickness. Nevertheless, a Zn gradient or Zn segregation towards the back cannot be fully excluded. The total sample compositions of the as-prepared CZTSe samples are shown in the quasi ternary phase diagram (Fig. 7).

Stoichiometric $\mathrm{Cu}_{2} \mathrm{ZnSnS}_{4}, \mathrm{Cu}_{2} \mathrm{ZnSnSe}_{4}, \mathrm{ZnS}$ and $\mathrm{ZnSe}$ powder reference samples are prepared by a solid-state reaction of the elements in sealed evacuated silica tubes at a maximum temperature of $750{ }^{\circ} \mathrm{C}$. Several annealing steps with subsequent mechanical grinding were performed to ensure chemical and structural homogeneity. The elemental composition was determined using wavelength dispersive X-ray fluorescence spectroscopy. Therefore a part of the powder was cast in epoxy and mechanically polished to yield a clean and very flat surface. ${ }^{32}$ Because of their exact stoichiometric composition and equilibration at high temperatures, these samples can be assumed to consist of single phases only.

Room temperature measurements of the X-ray absorption near edge structure at the K-edge of selenium at $12658 \mathrm{eV}$ were taken at Beamline $\mathrm{X}$ at the DORIS synchrotron at HASYLAB. ${ }^{33}$ Powder reference samples of $\mathrm{Cu}_{2} \mathrm{ZnSnSe}_{4}$ and $\mathrm{ZnSe}$ were ground and mixed with cellulose as a matrix, adjusting the edge step values of the X-ray absorption edge of selenium between one and two. Reference measurements were taken from pressed pellets in transmission configuration while measurements of thin film samples are taken in a total fluorescence yield setup using a PIPS detector. The effects of self-absorption on the spectra do not have to be taken into account since the thin film samples have a thickness of around $1 \mu \mathrm{m}$ while the calculated information depth on the high energy side of the Se K-edge in CZTSe extends to more than $15 \mu \mathrm{m} .{ }^{34}$ Therefore thin film samples can be treated as diluted samples at the Se K-edge. All Se K-edge spectra were measured using a Si(511)-monochromator in order to achieve the highest possible energy resolution. The intrinsic monochromator resolution of $0.11 \mathrm{eV}$ at $12.658 \mathrm{keV}$ is significantly broadened by the non-negligible vertical divergence of the beam. The spectra are further broadened by the limited lifetime of the core-hole in the Se 1 s-shell ${ }^{35}$ resulting in an effective energy resolution of about $2 \mathrm{eV}$ which is roughly two times worse compared to the effective energy resolution obtained at the K-edge of sulfur of about $1 \mathrm{eV} .{ }^{14}$ In order to be able to perform an accurate background subtraction of the continuous absorption background all spectra are measured within a range of $-230 \mathrm{eV}$ to $300 \mathrm{eV}$ with respect to the energy of the Se K-edge. An absolute calibration of the energy scale is not performed. Therefore the absolute energy scale might be inaccurate for some eV. All spectra of the K-edge of selenium are background subtracted fitting the background from $12606 \mathrm{eV}$ to $12646 \mathrm{eV}$ and are normalized using a post-edge region from $19.6 \mathrm{eV}$ to $97.4 \mathrm{eV}$ above the edge. Background subtraction, normalization and linear combination fitting of all spectra are performed with the help of the FEFF/IFEFFIT software package. ${ }^{36,37}$ 


\section{Results and discussion}

\section{Quantification of ZnSe in CZTSe thin films}

The measured, background-subtracted and edge-step normalized XANES spectra of powder references of $\mathrm{Cu}_{2} \mathrm{ZnSnSe}_{4}$ and $\mathrm{ZnSe}$ are compared in Fig. 1. Both spectra exhibit substantially distinct features and a significant edge shift of around $1.5 \mathrm{eV}$ with respect to each other. While in the case of $\mathrm{ZnSe}$ the maximum absorption appears at $12660 \mathrm{eV}$ directly above the edge, the spectrum of CZTSe is composed of a pre-edge feature at around $12656 \mathrm{eV}$

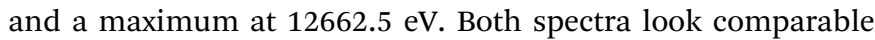
to their corresponding spectra at the K-edge of sulfur of the corresponding compounds CZTS and ZnS considering the features being broadened due to the lower energy resolution at the K-edge of Se. ${ }^{14}$

These substantial differences in the XANES spectra of $\mathrm{Cu}_{2} \mathrm{ZnSnSe}_{4}$ and $\mathrm{ZnSe}$ enable the quantification of $\mathrm{ZnSe}$ as a secondary phase in CZTSe samples. The measured XANES spectra of specific thin-film samples with varying $\mathrm{Zn} / \mathrm{Sn}$-ratios are shown in Fig. 1. It can be clearly seen that the pre-edge feature, characteristic for CZTSe, varies systematically with the $\mathrm{Zn} / \mathrm{Sn}$-ratio. The pre-edge feature growths with increasing CZTSe content, while the first maximum at $12660 \mathrm{eV}$ decreases according to the spectra being a superposition of the reference spectra. A linear combination analysis of a spectrum of one particular thin film sample is shown in Fig. 2. To be most sensitive to the distinct features of the reference spectra, the sample spectra are fitted within a range of $-3 \mathrm{eV}$ to $8 \mathrm{eV}$ with respect to the absorption edge position. The linear combination of the CZTSe and ZnSe reference spectra shows the best fit with $35.5 \%$ ZnSe and 64.5\% CZTSe and is in excellent agreement with the measured spectrum. Comparing two different spectra, the fits appear to be sensitive to a difference of roughly $3 \%$ in ZnSe-content. Nevertheless, the absolute error is slightly larger due to the choice of background subtraction and normalization parameters and is estimated to be around $5 \%$ absolute in ZnSe-content.

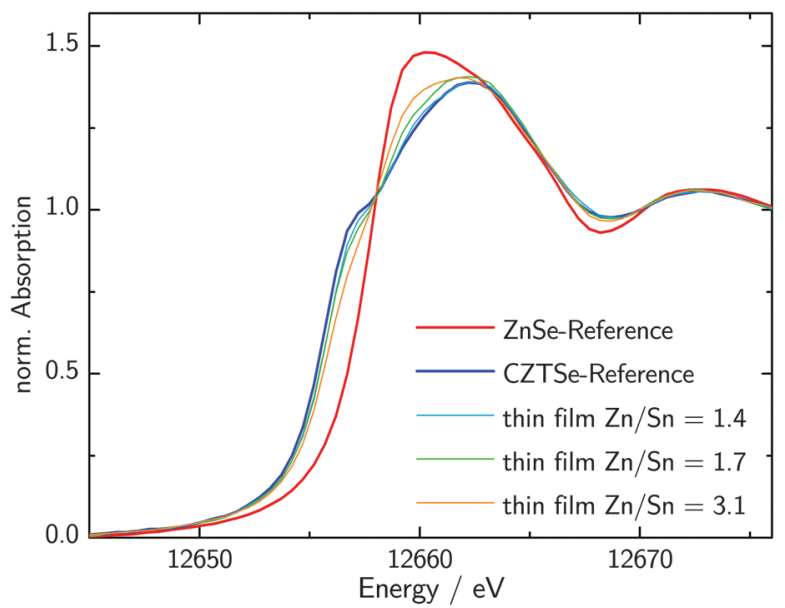

Fig. 1 Edge-step normalized XANES spectra of $\mathrm{Cu}_{2} \mathrm{ZnSnSe}_{4}$ and $\mathrm{ZnSe}$ measured at the K-edge of Se together with the spectra of CZTSe thin film samples with varying $\mathrm{Zn} / \mathrm{Sn}$-ratios.

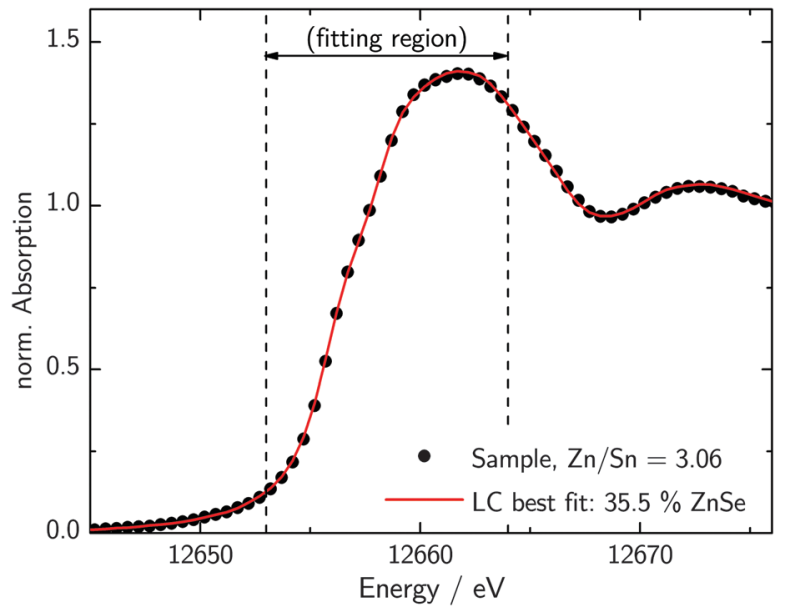

Fig. 2 Measured and edge-step normalized XANES spectrum of the Se K-edge of one specific thin film CZTSe sample (dotted) together with a fit obtained by a linear combination of CZTSe and ZnSe reference spectra (red).

\section{Principal stability of single phase CZTS/CZTSe}

Stoichiometric CZTS and CZTSe crystallize in the kesterite structure with 8 chalcogen atoms per unit cell. ${ }^{38,39}$ Correspondingly there are also 8 cations per unit cell, $4 \mathrm{Cu}, 2 \mathrm{Zn}$ and $2 \mathrm{Sn}$ atoms. Deviations of the stoichiometry can be described as combinations of intrinsic point defects which can be antisite defects, vacancies and interstitials. Due to the comparably high enthalpy of formation for interstitial point defects, ${ }^{22,40,41}$ it can be assumed that macroscopic deviations from stoichiometry can be attributed to antisite defects and vacancies only. Assuming a completely occupied anion lattice, the following constraint is found for the cations just by considering the limited number of lattice sites:

$$
\mathrm{S}=\mathrm{Cu}+\mathrm{Zn}+\mathrm{Sn}+\mathrm{V},
$$

where $\mathrm{V}$ denotes the number of cationic vacancies and $\mathrm{S}$ denotes the number of chalcogen atoms, sulfur or selenium. Note that because the atom count in eqn (1) is not site specific, antisites are included in the balance. If we assume the octet-rule to hold, which means that the sum of the cation valences equals the sum of the anion valences, a second constraint is found using:

$$
2 \mathrm{~S}=\mathrm{Cu}+2 \mathrm{Zn}+4 \mathrm{Sn} .
$$

Although it is conceivable that the octet rule is violated by the massive formation of defects or defect pairs which do not comply with eqn (2), we note that defects and defect clusters obeying this rule have been found in ab initio DFT calculations to exhibit significantly lower formation energies and are thus much more likely to occur. ${ }^{22,40,41}$ Note that only macroscopic deviations from stoichiometry in the range of several atomic percent are relevant for the herein presented analysis. The following analysis is based on the assumption of a complete anionic lattice (eqn (1)) as well as that macroscopic deviations from stoichiometry follow the concept of formal oxidation states (eqn (2)). Considering these two constraints for the atomic ratios in CZTS and CZTSe it follows that the $\mathrm{Zn} / \mathrm{Sn}$-ratio is not constrained and in principal can take any value, while the 
$\mathrm{Cu} / \mathrm{Sn}$-ratio is determined by the number of cationic vacancies in the material:

$$
\frac{\mathrm{Cu}}{\mathrm{Sn}}=2-\frac{2 \mathrm{~V}}{\mathrm{Sn}}
$$

Since the number of vacancies is always positive or zero, it follows that the $\mathrm{Cu} / \mathrm{Sn}$-ratio is constrained to be equal to or smaller than two.

\section{Composition of the CZTS/Se phase}

The total elemental sample composition as measured by XRF or EDX is a sum of compositions of the individual phases within a sample. For example, if we assume a sample to consist of CZTS, ZnS and $\mathrm{Cu}_{x} \mathrm{~S}$, the $\mathrm{Cu} / \mathrm{Sn}$ and $\mathrm{Zn} / \mathrm{Sn}$ ratios of the CZTS phase are significantly lower than the corresponding ratios of the total sample as a result of $\mathrm{Cu}$ and $\mathrm{Zn}$ being bound in secondary phases. By quantitatively measuring the phase composition of a sample and combining this information with the total composition of the overall sample it is therefore possible to recalculate the actual composition of the CZTS/ Se phase. The total sample compositions as determined by XRF in the case of CZTS and by EDX in the case of CZTSe are shown in Fig. 6 and 7, respectively. The given uncertainties are derived by Gaussian error propagation on the basis of a measurement uncertainty of 1 at\%. For the CZTS samples shown in Fig. 6, the total sample compositions are found to lie in the $\mathrm{Zn}$-rich and $\mathrm{Cu}$-rich regions of the quasi ternary phase diagram, whereas for the CZTSe samples shown in Fig. 7 the total sample compositions are found to be $\mathrm{Zn}$-rich located on the Cu-poor side of the $\mathrm{ZnSe}_{-} \mathrm{Cu}_{2} \mathrm{SnSe}_{3}$-tie line. These compositions are intentionally chosen to quantify $\mathrm{Cu}_{x} \mathrm{~S}$ and ZnS in CZTS while restricting the quantification to the ZnSe phase in CZTSe because of the limited spectral resolution at the Se K-edge.

To reveal the composition of the CZTS phase in samples with different $\mathrm{Cu} / \mathrm{Sn}$ and $\mathrm{Zn} / \mathrm{Sn}$-ratios, $\mathrm{ZnS}$ and $\mathrm{Cu}_{x} \mathrm{~S}$ are quantified by linear combination analysis of their XANES spectra as described elsewhere. ${ }^{14}$ ZnSe in CZTSe samples with different Zn/Sn-ratios is quantified as described above. The determined amounts of $\mathrm{ZnS}$, ZnSe and $\mathrm{Cu}_{x} \mathrm{~S}$ in CZTS/CZTSe thin films are shown in Fig. 3-5 with respect to the $\mathrm{Zn} / \mathrm{Sn}$ or $\mathrm{Cu} / \mathrm{Sn}$ ratio of the total sample composition. In each sample the quantity of formed secondary phases strongly correlates with the total sample composition. For example, in $\mathrm{Zn}$-rich samples $(\mathrm{Zn} / \mathrm{Sn}>1)$ the ratio of $\mathrm{ZnS} / \mathrm{ZnSe}$ correlates directly with the $\mathrm{Zn} / \mathrm{Sn}$-ratio, while for $\mathrm{Cu}$-rich samples with $\mathrm{Cu} / \mathrm{Sn}>2$ the secondary phase $\mathrm{Cu}_{x} \mathrm{~S}$ is formed.

To determine the amount of $\mathrm{Zn}$ which is consumed by $\mathrm{ZnS} /$ $\mathrm{ZnSe}$, it is important to note that a linear combination analysis of the chalcogen K-edge spectra directly determines the percentage $p_{\mathrm{X}}$ of chalcogen being bound in phase $\mathrm{X}$ with respect to the total amount of chalcogen atoms in the sample. The total amount of chalcogen in a CZTS/CZTSe sample is difficult to measure especially in the case of the sulfur compound due to the lack of reference samples and the strong matrix absorption of the $\mathrm{S}$ K-fluorescence lines. Instead the amount of chalcogen in the sample can be calculated from the metal composition taking the octet-rule into account, see eqn (2). In the following, the case of CZTS will be evaluated only, but all formulas also directly apply to the corresponding selenium containing compounds.

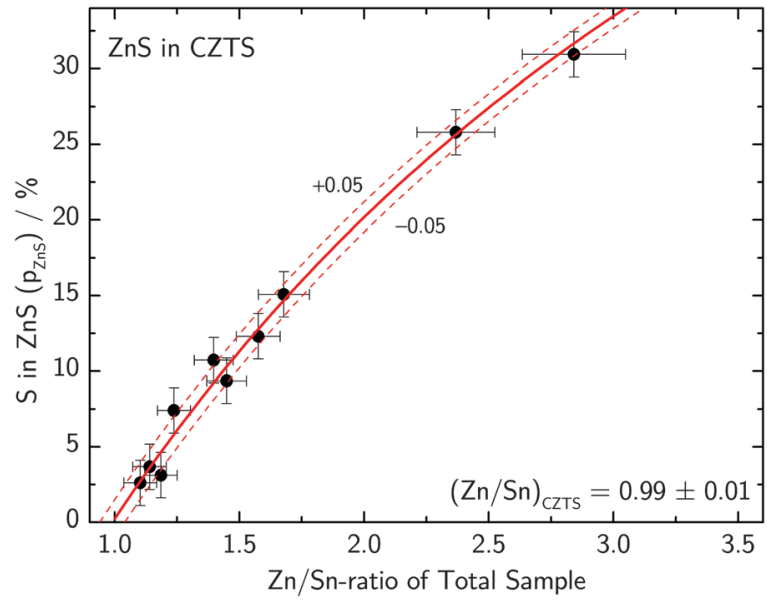

Fig. 3 Molar fraction of sulfur bound in ZnS in CZTS thin films with different $\mathrm{Zn} / \mathrm{Sn}$-ratios of the total sample composition as determined by $X A N E S$ analysis at the K-edge of sulfur together with a fit to eqn (5) which determines the $\mathrm{Zn} / \mathrm{Sn}$-ratio of the remaining CZTS phase.

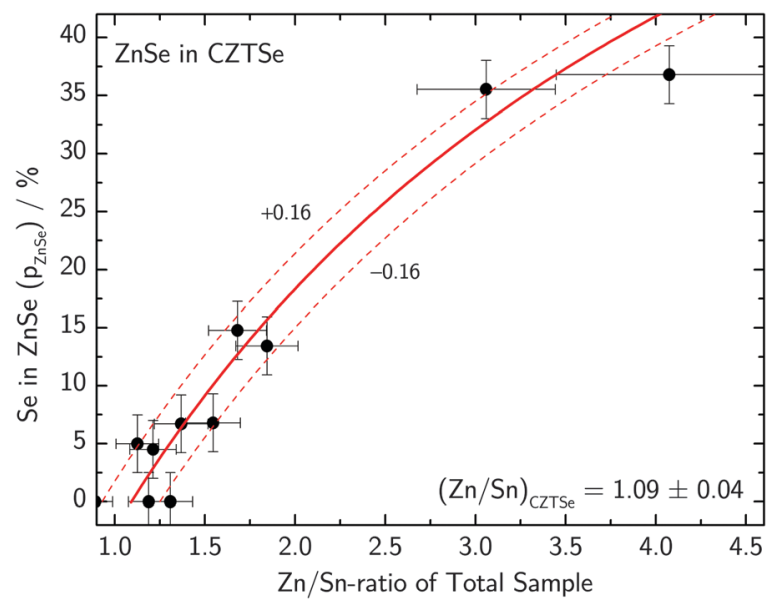

Fig. 4 Molar fraction of selenium bound in ZnSe in CZTSe thin films with different $\mathrm{Zn} / \mathrm{Sn}$-ratios of the total sample composition as determined by XANES analysis at the K-edge of selenium together with a fit to eqn (5) which determines the $\mathrm{Zn} / \mathrm{Sn}$-ratio of the remaining CZTSe phase.

Assuming $\mathrm{ZnS}$ to be in stoichiometric composition the amount of chalcogen in $\mathrm{ZnS}$ equals the amount of $\mathrm{Zn}$ in $\mathrm{ZnS}$. Therefore the amount of $\mathrm{Zn}$ in CZTS is given by:

$$
\mathrm{Zn}_{\mathrm{CZTS}}=\mathrm{Zn}_{\text {total }}-\mathrm{Zn}_{\mathrm{ZnS}}=\mathrm{Zn}_{\text {total }}-p_{\mathrm{Zns}} \cdot \mathrm{S}_{\text {total }},
$$

where element ${ }_{x}$ denotes the amount of a specific element in phase $\mathrm{X}$ or in the total sample, respectively.

Assuming a certain threshold composition of $\mathrm{Zn} / \mathrm{Sn}=Z$ above which all excess $\mathrm{Zn}$ forms $\mathrm{ZnS}$, it is possible to describe the formation of $\mathrm{ZnS}, p_{\mathrm{ZnS}}$, analytically by combining eqn (2) and (4):

$$
p_{\mathrm{ZnS}}=\frac{2\left(\frac{\mathrm{Zn}}{\mathrm{Sn}}-Z\right)}{C+\frac{2 \mathrm{Zn}}{\mathrm{Sn}}+4}
$$




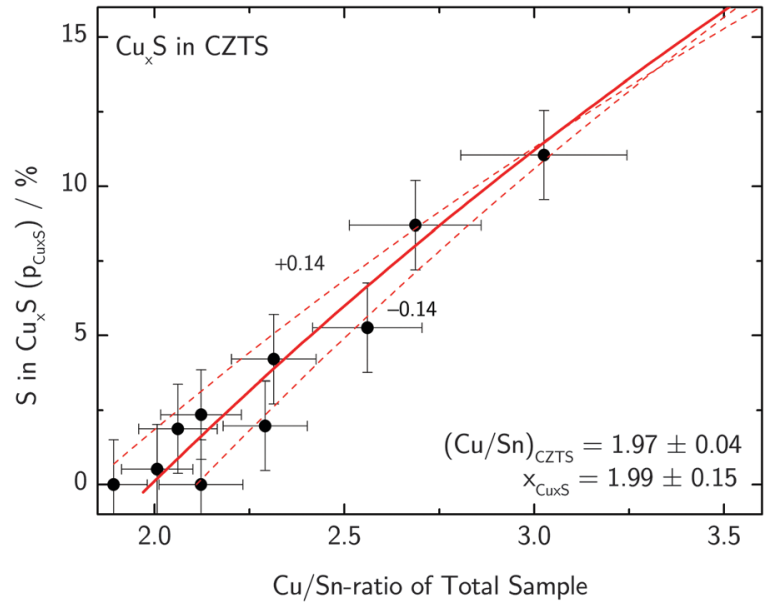

Fig. 5 Fraction of sulfur bound in $\mathrm{Cu}_{x} \mathrm{~S}$ found in CZTS thin films with different $\mathrm{Cu} / \mathrm{Sn}$-ratios of the total sample composition as determined by XANES analysis at the K-edge of sulfur together with a fit to eqn (7) which determines the $\mathrm{Cu} / \mathrm{Sn}$-ratio of the remaining CZTS phase.

where $C$ denotes the $\mathrm{Cu} / \mathrm{Sn}$-ratio. Applying eqn (2) assumes the octet-rule to be fulfilled for the total sample composition. Furthermore it is assumed that the only secondary phases forming are zinc sulfide and copper sulfide, while Sn is contained in CZTS only. Least square fits of this equation to the data for ZnS in CZTS and ZnSe in CZTSe are shown in Fig. 3 and 4, respectively. The formation of ZnS and $\mathrm{ZnSe}$ quite well follows this model description. Therefore the assumption of the existence of a certain $\mathrm{Zn} / \mathrm{Sn}$-ratio $Z$ above which all excess $\mathrm{Zn}$ forms $\mathrm{ZnS}$ seems to be confirmed by the experimental evidence. For CZTS this threshold value $Z$ is fitted to $Z_{\text {CZTS }}=$ $0.99 \pm 0.01$. This result implies that deviations from the stoichiometric composition $(\mathrm{Zn} / \mathrm{Sn}=1)$ immediately result in $\mathrm{ZnS}$ segregations. The given uncertainty is the error of the fit. The absolute error is larger due to the uncertainties of the XANES and XRF measurements. To estimate a value for the absolute error, upper and lower bounds of $Z$ are drawn in Fig. 3-5, such that the majority of the data points are considered within the given individual error bars. This procedure yields an absolute error for $Z_{\text {CZTS }}$ of \pm 0.05 , which is denoted in brackets in the following. The threshold value $Z$ for the formation of ZnSe in CZTSe samples is determined to be $Z_{\text {CZTSe }}=$ $1.09 \pm 0.04( \pm 0.16)$ which shows a tendency towards slightly Zn-rich compositions. Since this tendency is still in the order of the given uncertainties, it has to be interpreted carefully. From these measurements it can be concluded that already a slight $\mathrm{Zn}$ excess in the CZTS or CZTSe samples unavoidably leads to the segregation of $\mathrm{ZnS}$ or ZnSe as a secondary phase. Therefore it can be assumed that all devices fabricated in the $\mathrm{Zn}$-rich region of the phase diagram contain ZnS segregations. As a result, the composition of CZTS or CZTSe is constrained to $\mathrm{Zn} / \mathrm{Sn}=1$ for $\mathrm{Zn}$-rich growth conditions, as additionally shown in Fig. 6 and 7.

The measured amount of copper sulfide in the same CZTS sample series as above is shown in Fig. 5 with respect to the $\mathrm{Cu} / \mathrm{Sn}$-ratio of the sample. Note that for samples where $\mathrm{ZnS}$ was also found, the amount of $\mathrm{Zn}$ and $\mathrm{S}$ which segregated in $\mathrm{ZnS}$ was subtracted from the sample composition as described above, such that $p_{\mathrm{Cu}_{x} \mathrm{~S}}+p_{\mathrm{CZTS}}=100 \%$.

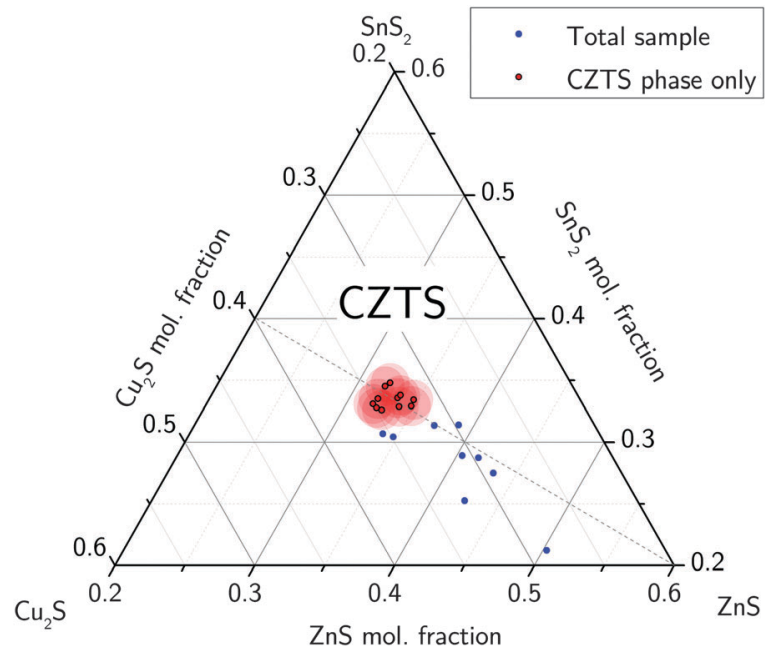

Fig. 6 Total sample compositions of CZTS thin films as measured by XRF (blue) together with the actual composition of the CZTS-phase (red) as determined by a combination of XRF and XANES analysis. The uncertainty of the CZTS phase composition is shown in slightly transparent red circles.

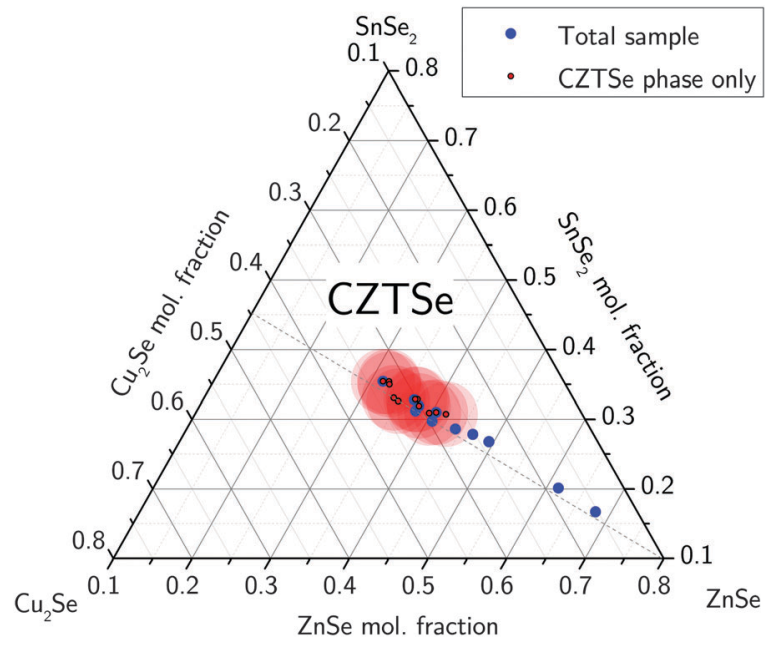

Fig. 7 Total sample compositions of CZTSe thin films as measured by EDX (blue) together with the actual composition of the CZTSe-phase (red) as determined by a combination of EDX and XANES analysis. The uncertainty of the CZTSe phase composition is shown in slightly transparent red circles.

The amount of $\mathrm{Cu}$ in the CZTS phase is given by subtracting the copper which is consumed by $\mathrm{Cu}_{x} \mathrm{~S}$ from the total amount of copper:

$$
\mathrm{Cu}_{\mathrm{CZTS}}=\mathrm{Cu}_{\text {total }}-\mathrm{Cu}_{\mathrm{Cu}_{x} \mathrm{~S}}=\mathrm{Cu}_{\text {total }}-(2-x) \cdot p_{\mathrm{Cu}_{x} \mathrm{~s}} \cdot \mathrm{S}_{\text {total }}
$$

Assuming a certain maximum compositional ratio $\mathrm{C}=\mathrm{Cu} / \mathrm{Sn}$ above which all excess copper will segregate in $\mathrm{Cu}_{x} \mathrm{~S}$, it is possible to find an analytical expression for the amount of copper sulfide formed $p_{\mathrm{Cu}_{x} \mathrm{~s}}$, dependent on the $\mathrm{Cu} / \mathrm{Sn}$-ratio of the sample by combining eqn (2) and (6):

$$
p_{\mathrm{Cu}_{x} \mathrm{~S}}=\frac{2 \frac{\mathrm{Cu}}{\mathrm{Sn}}-2 C}{x\left(\frac{\mathrm{Cu}}{\mathrm{Sn}}+2 Z+4\right)},
$$


with $Z=\mathrm{Zn} / \mathrm{Sn}=0.99$ as determined above. The only assumption which is made here, apart from the octet-rule to be fulfilled, is that a certain threshold value exists for the $\mathrm{Cu} / \mathrm{Sn}$-ratio. Since the value of $\mathrm{Cu} / \mathrm{Sn}$ is principally constrained by eqn (3), this assumption can be made. A least square fit of this expression to the measured data is shown in Fig. 5 and the threshold value of the $\mathrm{Cu} / \mathrm{Sn}$-ratio is determined to be $C=1.97 \pm 0.04( \pm 0.14)$. This value equals - within the experimental uncertainties - the theoretical value as determined by the above principal considerations. As a result, in Cu-rich CZTS thin films $(\mathrm{Cu} / \mathrm{Sn}>2)$ a $\mathrm{Cu}_{x} \mathrm{~S}$ phase segregates such that the $\mathrm{Cu} / \mathrm{Sn}$-ratio in the CZTS phase equals 2. On the other hand, for the most $\mathrm{Cu}$-poor sample, with $\mathrm{Cu} / \mathrm{Sn}=1.89(\mathrm{Zn} / \mathrm{Sn}=1.24)$ the only secondary phase detected by XANES was ZnS.

By subtracting copper and zinc which is segregated in the secondary phases $\mathrm{Cu}_{x} \mathrm{~S}$, ZnS or ZnSe from the composition of the total sample using eqn (4) and (6) it is now possible to calculate the actual composition of the CZTS/CZTSe phase. Total sample compositions as well as the composition of the CZTS/CZTSe phase revealed by this analysis are shown together in Fig. 6 and 7, respectively. The uncertainty related to the determination of the CZTS/CZTSe composition is also shown. It was calculated by error propagation of the uncertainties of the XRF, EDX and XANES measurements. The shown uncertainty is a mean value of individual uncertainties determined for all used CZTS/CZTSe samples, respectively. It clearly can be seen that within the uncertainty, the composition of the CZTS or CZTSe phase coincides with the point of stoichiometry as it was determined by the least square fitting above. Therefore it can be concluded that it is not possible to form neither Cu-rich nor Zn-rich CZTS even if grown under $\mathrm{Cu}$-rich or Zn-rich conditions, since in these cases excess copper or zinc will always segregate in secondary phases.

It has to be mentioned that it can be misleading to use the value of $\mathrm{Cu} /(\mathrm{Zn}+\mathrm{Sn})$ to determine the $\mathrm{Cu}$-richness of a sample, as is often done in the literature, because of the possible formation of ZnS. Most CZTS and CZTSe samples are synthesized in the $\mathrm{Zn}$-rich region of the phase diagram and therefore exhibit $\mathrm{ZnS}$ segregations, which reduce the Zn-content of the CZTS/Se phase. Therefore the CZTS phase can be stoichiometric in copper even if the $\mathrm{Cu} /(\mathrm{Zn}+\mathrm{Sn})$-value is below one. Therefore we propose to use the $\mathrm{Cu} / \mathrm{Sn}$-ratio and the $\mathrm{Zn} / \mathrm{Sn}$-ratio to describe the sample composition and compare it with various electrical characterizations, as the $\mathrm{Cu} / \mathrm{Sn}$-value is unaffected by the formation of $\mathrm{ZnS}$ and thus reflects, more directly, the composition of the CZTS/Se phase.

\section{Summary and conclusions}

It was shown that ZnSe as a secondary phase in CZTSe thin film samples can be identified and quantified using X-ray near edge absorption spectroscopy at the K-edge of selenium. ZnSe and CZTSe single phase references show distinct features in the $\mathrm{X}$-ray absorption near edge structure which enable the quantification of secondary phases in phase mixture systems by linear combination analysis. Due to a significant broadening of the absorption spectra at X-ray energies of the selenium K-edge, the quantification of $\mathrm{ZnSe}$ in CZTSe is not as precise as the quantification of ZnS in CZTS at the K-edge of sulfur, which has been demonstrated previously. ${ }^{14}$ We anticipate that with this method, $\mathrm{ZnSe}$ fractions down to at least $5 \%$ by volume can be quantified.

It was shown that the segregation of $\mathrm{ZnS}, \mathrm{ZnSe}$ or $\mathrm{Cu}_{x} \mathrm{~S}$ strongly correlates with the $\mathrm{Zn} / \mathrm{Sn}$ and $\mathrm{Cu} / \mathrm{Sn}$-ratio of the total sample composition.

By a combination of X-ray absorption spectroscopy at the chalcogen K-edges of CZTS/CZTSe and X-ray fluorescence it was demonstrated that it is possible to determine the actual composition of the CZTS/Se phase in mixed phase thin film samples. The determined composition of the CZTS/Se phase significantly deviates from the total sample composition. The $\mathrm{Zn} / \mathrm{Sn}$-ratio of the CZTS and CZTSe phases was shown to be limited to around one for the case of Zn-rich sample compositions. As a result, all excess $\mathrm{Zn}$ within the sample segregates in $\mathrm{ZnS} / \mathrm{ZnSe}$ as secondary phases.

Similarly, it was found that $\mathrm{Cu}_{x} \mathrm{~S}$ is formed in Cu-rich samples in such a way that the $\mathrm{Cu} / \mathrm{Sn}$-ratio of the CZTS phase is limited to two for Cu-rich total sample compositions. This shows that the single phase region of CZTS towards Cu-rich and Zn-rich compositions is rather small and coincides, within the measurement uncertainty, with the point of stoichiometry.

\section{Conflict of interest}

The authors declare no competing financial interest.

\section{Acknowledgements}

We gratefully acknowledge the provision of beamtime by HASYLAB as well as the support at the beamlines by Edmund Welter, Michael Murphy and Adam Webb. Additionally, the authors would like to thank Lars Steinkopf at the HZB for providing substrates and support during the deposition of the samples.

\section{References}

1 T. Unold and H. W. Schock, Nonconventional (Non-SiliconBased) Photovoltaic Materials, Annu. Rev. Mater. Res., 2011, 41, 297-321.

2 K. Ito, Copper Zinc Tin Sulfide-Based Thin Film Solar Cells, John Wiley \& Sons, 2015.

3 K. Ito and T. Nakazawa, Electrical and Optical Properties of Stannite-Type Quaternary Semiconductor Thin Films, Jpn. J. Appl. Phys., 1988, 27, 2094.

4 S. Chen, A. Walsh, J.-H. Yang, X. G. Gong, L. Sun, P.-X. Yang, J.-H. Chu and S.-H. Wei, Compositional dependence of structural and electronic properties of $\mathrm{CuZnSn}(\mathrm{S}, \mathrm{Se})$ alloys for thin film solar cells, Phys. Rev. B: Condens. Matter Mater. Phys., 2011, 83, 125201.

5 W. Wang, et al. Device Characteristics of CZTSSe Thin-Film Solar Cells with 12.6\% Efficiency, Adv. Energy Mater., 2014, 4, 1301465. 
6 C. Persson, Electronic and optical properties of $\mathrm{Cu}_{2} \mathrm{ZnSnS}_{4}$ and Cu2ZnSnSe4, J. Appl. Phys., 2010, 107, 053710.

7 D. M. Berg, et al. Discrimination and detection limits of secondary phases in $\mathrm{Cu}_{2} \mathrm{ZnSnS}_{4}$ using X-ray diffraction and Raman spectroscopy, Thin Solid Films, 2014, 569, 113-123.

$8 \mathrm{M}$. Ganchev, et al. Phase composition of selenized $\mathrm{Cu}_{2} \mathrm{ZnSnSe}_{4}$ thin films determined by X-ray diffraction and Raman spectroscopy, Thin Solid Films, 2011, 519, 7394-7398.

9 P. A. Fernandes, P. M. P. Salomé and A. F. da Cunha, Growth and Raman scattering characterization of $\mathrm{Cu}_{2} \mathrm{ZnSnS}_{4}$ thin films, Thin Solid Films, 2009, 517, 2519-2523.

10 A. Redinger, et al. Detection of a ZnSe secondary phase in coevaporated Cu2ZnSnSe4 thin films, Appl. Phys. Lett., 2011, 98, 101907.

11 E. A. Lund, H. Du, W. M. H. Oo, G. Teeter and M. A. Scarpulla, Investigation of combinatorial coevaporated thin film $\mathrm{Cu}_{2} \mathrm{ZnSnS}_{4}$ (II): Beneficial cation arrangement in Cu-rich growth, J. Appl. Phys., 2014, 115, 173503.

12 M. Grossberg, et al. Photoluminescence and Raman study of $\mathrm{Cu}_{2} \mathrm{ZnSn}(\mathrm{SexS} 1-x)_{4}$ monograins for photovoltaic applications, Thin Solid Films, 2011, 519, 7403-7406.

$13 \mathrm{X}$. Fontané, et al. In-depth resolved Raman scattering analysis for the identification of secondary phases: Characterization of $\mathrm{Cu}_{2} \mathrm{ZnSnS}_{4}$ layers for solar cell applications, Appl. Phys. Lett., 2011, 98, 181905.

14 J. Just, D. Lützenkirchen-Hecht, R. Frahm, S. Schorr and T. Unold, Determination of secondary phases in kesterite $\mathrm{Cu}_{2} \mathrm{ZnSnS}_{4}$ thin films by X-ray absorption near edge structure analysis, Appl. Phys. Lett., 2011, 99, 262105.

15 K. C. Mills, Thermodynamic data of Sulphides, Selenides and Tellurides, Butterworths, London, 1974.

16 B. Shin, et al. Thin film solar cell with $8.4 \%$ power conversion efficiency using an earth-abundant $\mathrm{Cu}_{2} \mathrm{ZnSnS}_{4}$ absorber, Prog. Photovoltaics, 2013, 21, 72-76.

17 I. Repins, et al. Co-evaporated $\mathrm{Cu}_{2} \mathrm{ZnSnSe}_{4}$ films and devices, Sol. Energy Mater. Sol. Cells, 2012, 101, 154-159.

18 J. J. Scragg, et al. Rapid annealing of reactively sputtered precursors for $\mathrm{Cu}_{2} \mathrm{ZnSnS}_{4}$ solar cells, Prog. Photovoltaics, 2014, 22, 10-17.

19 A. Redinger and S. Siebentritt, Coevaporation of $\mathrm{Cu}_{2} \mathrm{ZnSnSe}_{4}$ thin films, Appl. Phys. Lett., 2010, 97, 092111.

20 H. Katagiri, K. Jimbo, M. Tahara, H. Araki and K. Oishi, The influence of the composition ratio on CZTS-based thin film solar cells, Mater. Res. Soc. Symp. Proc. 2009, 1165, M04-01.

21 V. Kosyak, M. A. Karmarkar and M. A. Scarpulla, Temperature dependent conductivity of polycrystalline $\mathrm{Cu}_{2} \mathrm{ZnSnS}_{4}$ thin films, Appl. Phys. Lett., 2012, 100, 263903.

22 S. Chen, A. Walsh, X.-G. Gong and S.-H. Wei, Classification of Lattice Defects in the kesterite $\mathrm{Cu}_{2} \mathrm{ZnSnS}_{4}$ and $\mathrm{Cu}_{2} \mathrm{ZnSnSe}_{4}$ Earth-Abundant Solar Cell Absorbers, Adv. Mater., 2013, 25, 1522-1539.

23 I. D. Olekseyuk, I. V. Dudchak and L. V. Piskach, Phase equilibria in the $\mathrm{Cu}_{2} \mathrm{~S}-\mathrm{ZnS}-\mathrm{SnS}_{2}$ system, J. Alloys Compd., 2004, 368, 135-143.

24 A. Lafond, L. Choubrac, C. Guillot-Deudon, P. Deniard and S. Jobic, Crystal Structures of Photovoltaic Chalcogenides, an Intricate Puzzle to Solve: the Cases of CIGSe and CZTS Materials, Z. Anorg. Allg. Chem., 2012, 638, 2571-2577.

25 L. Choubrac, A. Lafond, C. Guillot-Deudon, Y. Moëlo and S. Jobic, Structure Flexibility of the $\mathrm{Cu}_{2} \mathrm{ZnSnS}_{4}$ Absorber in Low-Cost Photovoltaic Cells: From the Stoichiometric to the Copper-Poor Compounds, Inorg. Chem., 2012, 51, 3346-3348.

26 B.-A. Schubert, et al. $\mathrm{Cu}_{2} \mathrm{ZnSnS}_{4}$ thin film solar cells by fast coevaporation, Prog. Photovoltaics, 2011, 19, 93-96.

27 V. A. Solé, E. Papillon, M. Cotte, P. Walter and J. Susini, A multiplatform code for the analysis of energy-dispersive X-ray fluorescence spectra, Spectrochim. Acta, Part B, 2007, 62, 63-68.

28 T. Ericson, T. Kubart, J. J. Scragg and C. Platzer-Björkman, Reactive sputtering of precursors for $\mathrm{Cu}_{2} \mathrm{ZnSnS}_{4}$ thin film solar cells, Thin Solid Films, 2012, 520, 7093-7099.

29 E. Welter, Beamline A1 - Hasylab. AIP Conf Proc 955, 2010.

30 W. Ki and H. W. Hillhouse, Earth-Abundant Element Photovoltaics Directly from Soluble Precursors with High Yield Using a Non-Toxic Solvent, Adv. Energy Mater., 2011, 1, 732-735.

31 C. M. Sutter-Fella, et al. Sodium Assisted Sintering of Chalcogenides and Its Application to Solution Processed $\mathrm{Cu}_{2} \mathrm{ZnSn}(\mathrm{S}, \mathrm{Se})_{4}$ Thin Film Solar Cells, Chem. Mater., 2014, 26, 1420-1425.

32 S. Schorr, H.-J. Hoebler and M. Tovar, A neutron diffraction study of the stannite-kesterite solid solution series, Eur. J. Mineral., 2007, 19, 65-73.

33 R. Frahm, Beamline X - Hasylab, Rev. Sci. Instrum., 1989, 60, 2515-2518.

34 B. L. Henke, E. M. Gullikson and J. C. Davis, X-ray interactions: photoabsorption, scattering, transmission, and reflection at $E=50-30000 \mathrm{eV}, Z=1-92$, At. Data Nucl. Data Tables, 1993, 54, 181-342.

35 M. O. Krause and J. H. Oliver, Natural widths of atomic K and L levels, $\mathrm{K} \alpha \mathrm{X}$-ray lines and several KLL Auger lines, J. Phys. Chem. Ref. Data, 1979, 8, 329-338.

36 M. Newville, IFEFFIT: interactive XAFS analysis and FEFF fitting, J. Synchrotron Radiat., 2001, 8, 322-324.

37 B. Ravel and M. Newville, ATHENA, ARTEMIS, HEPHAESTUS: data analysis for X-ray absorption spectroscopy using IFEFFIT, J. Synchrotron Radiat., 2005, 12, 537-541.

38 S. R. Hall, J. T. Szymanski and J. M. Stewart, Kesterite, $\mathrm{Cu}_{2}(\mathrm{Zn}, \mathrm{Fe}) \mathrm{SnS}_{4}$, and stannite, structurally similar but distinct minerals, Can. Mineral., 1978, 16, 131-137.

39 S. Schorr, The crystal structure of kesterite type compounds: A neutron and X-ray diffraction study, Sol. Energy Mater. Sol. Cells, 2011, 95, 1482-1488.

40 S. Chen, J.-H. Yang, X. G. Gong, A. Walsh and S.-H. Wei, Intrinsic point defects and complexes in the quaternary kesterite semiconductor $\mathrm{Cu}_{2} \mathrm{ZnSnS}_{4}$, Phys. Rev. B: Condens. Matter Mater. Phys., 2010, 81, 245204.

41 D. Han, et al. Deep electron traps and origin of p-type conductivity in the earth-abundant solar-cell material $\mathrm{Cu}_{2} \mathrm{ZnSnS}_{4}$, Phys. Rev. B: Condens. Matter Mater. Phys., 2013, 87, 155206. 